\title{
Avaliação da funcionalidade de idosos institucionalizados: relação entre a MIF e a ICF
}

\author{
Evaluation of the functionality of institutionalized elderly people: \\ relation between the functional independence measure (FIM) and the \\ international classification of functioning, disability and health (ICF)
}

\author{
Tânia Cristina Malezan Fleig, ${ }^{1}$ Murilo Rezende Oliveira² \\ 'Universidade de Santa Cruz do Sul (Unisc), Santa Cruz do Sul, RS, Brasil. \\ ZUniversidade Federal de Santa Maria (UFSM), Santa Maria, RS, Brasil.
}

Recebido em: 26/01/2016 / Aceito em: 24/05/2017 / Publicado em: 30/06/2017

murilorezendeoliveira@hotmail.com

\section{RESUMO}

Objetivo: demonstrar através da Medida de Independência Funcional (MIF), as características funcionais de idosos em instituições de longa permanência para idosos (ILPI's), identificando a possível relação deste instrumento com a Classificação Internacional de Funcionalidade, Incapacidade e Saúde (ICF). Método: trata-se de um estudo transversal, com 55 idosos institucionalizados com idade média de 79,7 $\pm 10,2$ anos. A capacidade funcional foi avaliada através da MIF, sendo os domínios deste instrumento relacionados com as categorias da ICF. Cada idoso foi qualificado, quanto às limitações funcionais conforme a ICF. A análise de dados foi descrita em média, desvio padrão e frequência. Resultados: $72,7 \%$ dos idosos eram do sexo feminino, sendo a doença de Alzheimer o diagnóstico mais observado $(45,4 \%)$. No que diz respeito à capacidade funcional, houve um predomínio de idosos com independência completa (47,3\%). Na relação das categorias da ICF, com os domínios do instrumento de avaliação funcional, mostrou-se positiva em todos os itens. Considerações finais: os resultados indicam que os idosos em questão apresentavam independência completa para realizar suas atividades de vida diária. Além disso, a relação do instrumento de avaliação funcional com a ICF possibilitou-nos uma visão ampliada sobre o processo de envelhecimento de uma população de idosos institucionalizados, principalmente no componente Atividade e Participação, podendo ser utilizada como indicadora para o desenvolvimento de estratégias e propostas de intervenções que favoreçam a demanda clínica e pessoal destes idosos.

Palavras-chave: Idoso; Institucionalização; Classificação Internacional de Funcionalidade; Incapacidade e Saúde.

\section{ABSTRACT}

Objective: to demonstrate, through the Functional Independence Measure (FIM), the functional characteristics of the elderly in long-term care institutions for elderly, identifying the possible relationship between this instrument and the International Classification of Functioning, Disability and Health (ICF). Method: this is a cross-sectional study with 55 institutionalized elderly with mean age of $79.7 \pm 10.2$ years. Functional capacity was assessed through FIM, and the domains of this instrument are related to the categories of ICF. Each elderly person was qualified for functional limitations according to ICF. Data analysis was described on average, standard deviation and frequency. Results: $72.7 \%$ of the elderly were female, with Alzheimer's disease being the most observed diagnosis (45.4\%). Regarding functional capacity there was a predominance of elderly individuals with complete independence (47.3\%). The relation between the ICF categories and the domains of the functional evaluation instrument was positive in all items. Closing remarks: the results indicate that the elderly in question presented complete independence to perform their daily life activities. In addition, the relationship between the functional assessment instrument and the ICF enabled us to gain a broader view of the aging process of a population of institutionalized elder- 
ly, mainly in the activity and participation, and can be used as an indicator for the development of strategies and proposals for interventions that favor the clinical and personal demands of these elderly people.

Keywords: Aged; Institutionalization; International Classification of Functioning; Disability and Health.

\section{INTRODUCÃO}

É observado, nos últimos anos, um aumento expressivo de idosos na população mundial e também no Brasil. Tal condição pode ser explicada pela articulação entre os mecanismos que reduzem a mortalidade e a diminuição dos índices de natalidade de uma população, configurando o que se denomina transição demográfica. ${ }^{1}$ Também explica-se devido aos avanços no campo da saúde, acompanhado por mudanças nas estruturas e nos papéis da família, bem como nos padrões de trabalho e migração, havendo modificação do perfil de morbimortalidade, ${ }^{2}$ ocorrendo, necessariamente, um desenvolvimento de pesquisas na área de Gerontologia e Geriatria. ${ }^{3}$

O processo de transição demográfica exige transformações sociais e geram um aumento na demanda de instituições de longa permanência para idosos (ILPI) no país. Tornando-se, essas instituições, uma alternativa importante de assistência, devendo assegurar qualidade de vida e satisfação aos idosos atendidos, como também para suas famílias. ${ }^{4}$

O idoso que é institucionalizado, na maioria das vezes, distancia-se do convívio familiar, da própria casa e dos amigos, contribuindo para a perda de sua autonomia e dificultando a elaboração de novos projetos. Essa exclusão social pode estar associada às sequelas de doenças crônicas não-transmissíveis que são uma das principais causas das admissões em instituições. Tais sequelas frequentemente causam prejuízos funcionais que tornam os idosos dependentes de cuidados especiais. ${ }^{4}$ Logo, o processo de avaliação da capacidade funcional e o reconhecimento da funcionalidade do idoso, tornam-se essenciais para o estabelecimento de um diagnóstico e um prognóstico que servirão de base para as decisões sobre os cuidados necessários às pessoas idosas. Sendo um parâmetro que, juntamente a outros indicadores de saúde, poderá definir ações que irão resultar na efetividade e a eficiência das intervenções propostas. ${ }^{5}$

Nesse contexto, a Classificação Internacional de Funcionalidade, Incapacidade e Saúde (ICF) é uma proposta pela Organização Mundial de Saúde (OMS) que foi adotada por Portugal, na qual são atribuídas diversas vantagens de utilização. Segundo Quintana et al., ${ }^{3}$ esta classificação tem como propósito o foco na capacidade das pessoas, envolvendo demandas biológicas, psicológicas e sociais e não somente as questões de incapacidade do indivíduo. Sendo uma abordagem relativamente nova, pode oferecer uma válida e confiável base para a identificação de problemas de saúde relevantes. ${ }^{6}$

Dentre as diversas escalas existentes para avaliar a capacidade funcional dos idosos institucionalizados, tem-se a Medida de Independência Funcional (MIF). Tal instrumento é usado para medir o grau de cuidado que a pessoa, com deficiência, requer para executar atividades motoras e cognitivas. ${ }^{7}$

Dessa forma, o objetivo deste estudo é demons- trar, através da MIF, as características funcionais de idosos em instituições de longa permanência (ILPI's) e relacionar tal instrumento de avaliação funcional à Classificação Internacional de Funcionalidade, Incapacidade e Saúde (ICF) para reconhecimento da abordagem fisioterapêutica.

\section{METODOLOGIA}

Trata-se de um estudo observacional descritivo, com caráter transversal, de natureza quantitativa e analítica. ${ }^{8}$ A pesquisa foi aprovada pelo Comitê de Ética em Pesquisa da Universidade de Santa Cruz do Sul UNISC, segundo critérios estabelecidos pela resolução CNS/MS 466/12, com número do parecer: 1.378.449.

A coleta de dados foi realizada em uma Instituição de Longa Permanência para Idosos (ILPI), de caráter particular, onde residiam 63 idosos, na cidade de Cachoeira do Sul - RS, no período de janeiro e fevereiro de 2016.

Os seguintes critérios de inclusão foram considerados: idade igual ou superior a 60 anos; idosos de ambos os sexos e seus respectivos cuidadores que consentiram com a participação por meio do Termo de Consentimento Livre e Esclarecido (TCLE). Os critérios de exclusão adotados foram idosos acamados e aqueles que não concordaram com o TCLE, assim como seus cuidadores.

A amostra foi composta por 63 pacientes idosos, sendo que 8 foram excluídos por serem acamados, totalizando 55 idosos e seus respectivos cuidadores. Tal amostra foi selecionada por conveniência de acesso do acadêmico pesquisador ao local e, consequentemente, aos sujeitos.

Para a coleta das características clínicas e sociodemográficas foi elaborado e preenchido pelos pesquisadores um questionário, com as seguintes perguntas: idade, sexo e diagnóstico médico.

Além disso, foram identificados os níveis de alfabetização dos idosos, segundo o teste de cognição denominado Mini Exame de Estado Mental (MEEM). O MEEM é utilizado para detectar os níveis de alfabetização dos idosos e identificar a presença de algum déficit cognitivo não diagnosticado. ${ }^{9}$ De acordo com Lourenço e Veras, o MEEM é composto por questões agrupadas em 7 categorias: orientação de tempo (5 pontos), lembrança de palavras (3 pontos), atenção e cálculo (5 pontos), registro de 3 palavras (3 pontos), capacidade construtiva visual (1 ponto). O escore varia de 0 a 30 pontos com tempo de aplicação de 5 a 10 minutos. A pontuação é dada de acordo com a escolaridade da pessoa avaliada, sendo considerado com uma possível demência aqueles, com escolaridade superior a 11 anos, que pontue um valor menor que 24; já, aqueles que tiverem escolaridade entre 1 e 11 anos, serão considerados com possível demência quando pontuarem menos que 18 e os analfabetos deverão pontuar menos que 14. Tais informações foram coletadas diretamente com os idosos pelo pesquisador responsável. ${ }^{10}$

Dentre as diversas escalas existentes para avaliar a capacidade funcional dos idosos institucionalizados, tem-se a Medida de Independência Funcional (MIF). A MIF é organizada pela classificação do paciente em sua habilidade para executar uma atividade e a sua necessidade por assistência de outra pessoa ou recurso de adaptação. Se a ajuda é necessária, a escala quantifica 
essa necessidade. É dividida em categorias com um total de 8 itens e 2 dimensões subdivididas, definidos para avaliar a independência do indivíduo, em realizar, de modo satisfatório e eficaz, as atividades básicas. Estas atividades incluem o mínimo de habilidade para as categorias de auto cuidado, controle do esfíncter, transferência e locomoção (dimensão motora), comunicação e cognitivo social (dimensão cognitiva). Nesse instrumento, o escore inclui 7 pontos, representando o nível de completa independência e o 1, completa dependência. O escore total na escala MIF é calculado a partir da soma de pontos atribuídos a cada item dentro das categorias. ${ }^{11}$ Observando-se que, quanto menor o escore da escala, maior será a deficiência.

Logo, as informações sobre a capacidade funcional destes idosos eram preenchidas individualmente com o seu respectivo cuidador, por motivos de reconhecimento das atividades e da capacidade que os idosos eram capazes de realizar no seu dia-a-dia. Ressalva-se que os cuidadores foram treinados para preencher a escala com o mesmo pesquisador responsável, para que não houvesse qualquer intervenção do mesmo nas respostas. Com tais dados obtidos, o pesquisador relacionou-os com a ICF.

A ICF estrutura a informação de forma integrada e simples em duas partes: a primeira parte diz respeito à Funcionalidade e Incapacidade e a segunda aos Fatores Contextuais. Cada uma das partes subdivide-se ainda em dois componentes. Os componentes da parte Funcionalidade e Incapacidade são: Funções e Estruturas do corpo e Atividades e Participação. Os Fatores Contextuais são compostos pelas componentes Fatores Ambientais e Pessoais. As unidades de classificação da ICF são as categorias dentro dos domínios da saúde e daqueles relacionados com a saúde. Cada categoria da ICF tem atribuído um código, composto por uma letra que se refere aos componentes da classificação (b: funções do corpo, $s$ : estruturas do corpo, $d$ : atividades e participação e, e: fatores ambientais), seguido de um código numérico, iniciado pelo número do capítulo (um dígito), seguido do segundo nível (dois dígitos) e do terceiro e quarto níveis (um dígito cada)..$^{12}$

Para avaliação da concordância entre a MIF e a ICF, foi necessário realizar uma correlação prévia das pontuações das escalas, assim como Fréz et al. (2013) ${ }^{7}$ usou em seu estudo, considerando respectivamente: independência completa e independência modificada = nenhuma dificuldade (qualificador 0); supervisão e dependência mínima = dificuldade leve (qualificador 1); dependência moderada = dificuldade moderada (qualificador 2); dependência máxima = dificuldade grave(qualificador 3 ) e dependência total = dificuldade completa (qualificador 4). Foi seguido tal modelo pelo fato de que esta escala é amplamente reconhecida e utilizada no dia-a-dia de muitos profissionais, necessitando assim uma possível relação com a ICF para que, quando comparadas, possam ser quantificadas de maneira mais válida possível.

A análise de dados das características clínicas e sociodemográficas, da MIF, do MEEM e dos qualificadores da ICF foi realizada por meio do programa SPSS (versão 20.0), sendo os dados expressos em frequência, média e desvio padrão.

\section{RESULTADOS}

A amostra foi composta por 55 idosos com média de idades de 79,7 $\pm 10,21$ anos, sendo suas características clínicas e sociodemográficas descritas na tabela 1. Tais dados foram obtidos através da observação de prontuários individuais fornecidos pela ILPI. Prontuários estes que foram preenchidos por enfermeiros e médicos responsáveis pela instituição baseados em depoimentos de familiares e exames médicos. Além disso, na tabela 1 está representado o nível de escolaridade avaliada pelo MEEM.

Tabela 1 - Características clínicas e sociodemográficas dos idosos institucionalizados.

\begin{tabular}{lc}
\hline Variáveis & $\mathbf{n}(\%)$ \\
\hline Sexo & $40(72,7)$ \\
Feminino & $15(27,3)$ \\
Masculino & \\
Diagnóstico & $25(45,5)$ \\
Alzheimer & $8(14,5)$ \\
AVE & $5(9,1)$ \\
Depressão & $4(7,3)$ \\
Parkinson & $4(7,3)$ \\
Artroplastia Total de Quadril & $3(5,5)$ \\
DPOC & $3(5,5)$ \\
Artrose & $2(3,6)$ \\
Artrite Reumatóide & $1(1,8)$ \\
Artroplastia total de Joelho & \\
Escolaridade & $25(45,4)$ \\
Analfabeto & $7(12,7)$ \\
1 - 11 anos & $23(41,8)$ \\
>11 anos &
\end{tabular}

Dados expressos em frequência. AVE: Acidente Vascular Encefálico; DPOC: Doença Pulmonar Obstrutiva Crônica.

Na tabela 2, tem-se a classificação da capacidade funcional, segundo a Medida de Independência Funcional (MIF), dos idosos institucionalizados.

Tabela 2 - Classificação da capacidade funcional dos idosos institucionalizados de acordo com a MIF.

\begin{tabular}{lc}
\hline Escore & n (\%) \\
\hline MIF & $26(47,3)$ \\
Independência Completa & $13(23,6)$ \\
Dependência Mínima & $9(16,4)$ \\
Dependência Moderada & $7(12,7)$ \\
Dependência Máxima & \\
\hline
\end{tabular}

Dados expressos em frequência.

$\mathrm{Na}$ tabela 3 estão representadas as relações das categorias da ICF, com cada componente da MIF.

A relação das categorias da MIF está para o segundo qualificador de atividade e participação que corresponde a capacidade (sem ajuda). Dados expressos em frequência. 
Tabela 3 - Categorias da ICF selecionadas para cada componente da Medida de Independência Funcional (MIF).

\begin{tabular}{|c|c|c|c|c|c|c|c|}
\hline MIF & $\begin{array}{l}\text { Categoria } \\
\text { ICF }\end{array}$ & Descrição & $\begin{array}{c}\text { Nenhuma } \\
\text { dificuldade } \\
\text { n (\%) }\end{array}$ & $\begin{array}{c}\text { Dificuldade } \\
\text { leve } \\
n(\%)\end{array}$ & $\begin{array}{c}\text { Dificuldade } \\
\text { Moderada } \\
\text { n (\%) }\end{array}$ & $\begin{array}{c}\text { Dificuldade } \\
\text { grave } \\
\text { n (\%) }\end{array}$ & $\begin{array}{c}\text { Dificuldade } \\
\text { Completa } \\
\text { n (\%) }\end{array}$ \\
\hline \multicolumn{8}{|l|}{ CUIDADO PESSOAL } \\
\hline Alimentar-se & d550 & Comer & $34(61,8)$ & $14(25,5)$ & $5(9,1)$ & $2(3,6)$ & - \\
\hline Arrumar-se & d560 & Beber & $34(61,8)$ & $14(25,5)$ & $5(9,1)$ & $2(3,6)$ & - \\
\hline Banhar-se & d520 & Cuidados das partes do corpo & $23(41,8)$ & $9(16,4)$ & $6(10,9)$ & $17(30,9)$ & - \\
\hline Vestir-se. Parte Superior e & d510 & Lavar-se & $16(29,1)$ & $15(27,3)$ & $5(9,1)$ & $14(25,5)$ & $5(9,1)$ \\
\hline Inferior & d540 & Vestir-se & $22(40,0)$ & $11(20,0)$ & $9(16,4)$ & $13(23,6)$ & - \\
\hline Higiene Pessoal & d520 & Cuidados das partes do corpo & $23(41,8)$ & $9(16,4)$ & $6(10,9)$ & $17(30,9)$ & - \\
\hline \multicolumn{8}{|c|}{ CONTROLE ESFINCTERIANO } \\
\hline \multirow[t]{2}{*}{ Controle Vesical } & b620 & Funções Urinárias & $20(36,4)$ & $12(21,8)$ & $6(10,9)$ & $10(18,2)$ & $7(12,7)$ \\
\hline & d5300 & Regulação da micção & $20(36,4)$ & $12(21,8)$ & $6(10,9)$ & $10(18,2)$ & $7(12,7)$ \\
\hline \multirow[t]{2}{*}{ Controle Intestinal } & b525 & Funções de defecação & $26(47,3)$ & $10(18,2)$ & $3(5,5)$ & $9(16,4)$ & $7(12,7)$ \\
\hline & d5301 & Regulação da defecação & $26(47,3)$ & $10(18,2)$ & $3(5,5)$ & $9(16,4)$ & $7(12,7)$ \\
\hline \multicolumn{8}{|l|}{ MOBILIDADE } \\
\hline \multirow[t]{2}{*}{ Transferência } & $\mathrm{d} 410$ & Mudar a posição básica do corpo & $27(49,1)$ & $7(12,7)$ & $7(12,7)$ & $11(20,0)$ & $3(5,5)$ \\
\hline & d420 & Transferir a própria posição & $31(56,4)$ & $9(16,4)$ & $6(10,9)$ & $8(14,5)$ & $1(1,8)$ \\
\hline \multirow[t]{2}{*}{ Sanitário } & $\mathrm{d} 410$ & Mudar a posição básica do corpo & $27(49,1)$ & $7(12,7)$ & $7(12,7)$ & $11(20,0)$ & $3(5,5)$ \\
\hline & d420 & Transferir a própria posição & $31(56,4)$ & $9(16,4)$ & $6(10,9)$ & $8(14,5)$ & $1(1,8)$ \\
\hline \multirow[t]{2}{*}{ Banheiro, Chuveiro } & $\mathrm{d} 410$ & Mudar a posição básica do corpo & $27(49,1)$ & $7(12,7)$ & $7(12,7)$ & $11(20,0)$ & $3(5,5)$ \\
\hline & d420 & Transferir a própria posição & $31(56,4)$ & $9(16,4)$ & $6(10,9)$ & $8(14,5)$ & $1(1,8)$ \\
\hline \multicolumn{8}{|l|}{ LOCOMOC̣ÃO } \\
\hline \multirow[t]{4}{*}{ Marcha/Cadeira de Rodas } & d450 & Andar & $21(37,8)$ & $9(16,4)$ & $12(21,8)$ & $6(10,9)$ & - \\
\hline & d455 & Deslocar-se & $21(37,8)$ & $9(16,4)$ & $12(21,8)$ & $6(10,9)$ & - \\
\hline & d460 & Deslocar-se por diferentes locais & $21(37,8)$ & $9(16,4)$ & $12(21,8)$ & $6(10,9)$ & - \\
\hline & d465 & $\begin{array}{l}\text { Deslocar-se usando algum tipo de } \\
\text { equipamento }\end{array}$ & - & - & - & $3(5,4)$ & $4(7,2)$ \\
\hline \multirow[t]{2}{*}{ Escadas } & e120 & $\begin{array}{l}\text { Produto e tecnologia para mobilidade } \\
\text { e transporte pessoal na vida diária }\end{array}$ & - & - & - & $3(5,4)$ & $4(7,2)$ \\
\hline & d455 & Deslocar-se & $16(29,1)$ & $15(27,3)$ & $4(7,3)$ & $16(28,8)$ & $4(7,2)$ \\
\hline \multicolumn{8}{|l|}{ COMUNICAC̣ÃO } \\
\hline \multirow[t]{3}{*}{ Abrangência oral/visual } & b156 & Funções de percepção & $30(54,5)$ & $12(21,8)$ & $9(16,4)$ & $4(7,3)$ & - \\
\hline & d310 & $\begin{array}{l}\text { Comunicação-recepção de mensa- } \\
\text { gens orais }\end{array}$ & $30(54,5)$ & $12(21,8)$ & $9(16,4)$ & $4(7,3)$ & - \\
\hline & d325 & $\begin{array}{l}\text { Comunicação-recepção de mensa- } \\
\text { gens escritas }\end{array}$ & $28(50,9)$ & $18(32,7)$ & $5(9,1)$ & $4(7,3)$ & - \\
\hline \multirow[t]{7}{*}{ Expressão Vocal/Não Verbal } & b167 & Funções mentais de linguagem & $30(54,5)$ & $12(21,8)$ & $9(16,4)$ & $4(7,3)$ & - \\
\hline & b320 & Funções de articulação & $30(54,5)$ & $12(21,8)$ & $9(16,4)$ & $4(7,3)$ & - \\
\hline & d310 & $\begin{array}{l}\text { Comunicação-recepção de mensa- } \\
\text { gens orais }\end{array}$ & $30(54,5)$ & $12(21,8)$ & $9(16,4)$ & $4(7,3)$ & - \\
\hline & d315 & $\begin{array}{l}\text { Comunicação-recepção de mensa- } \\
\text { gens não verbais }\end{array}$ & $30(54,5)$ & $12(21,8)$ & $9(16,4)$ & $4(7,3)$ & - \\
\hline & d330 & Fala & $30(54,5)$ & $12(21,8)$ & $9(16,4)$ & $4(7,3)$ & - \\
\hline & d335 & Produção de mensagens não verbais & $30(54,5)$ & $12(21,8)$ & $9(16,4)$ & $4(7,3)$ & - \\
\hline & d360 & $\begin{array}{l}\text { Utilização de dispositivos e técnicas } \\
\text { de comunicação }\end{array}$ & - & - & - & - & - \\
\hline \multicolumn{8}{|l|}{ CONHECIMENTO SOCIAL } \\
\hline \multirow[t]{2}{*}{ Interação Social } & d710 & Interações interpessoais básicas & $21(38,2)$ & $15(27,3)$ & $14(25,5)$ & $4(7,3)$ & $1(1,8)$ \\
\hline & d720 & Interações interpessoais complexas & $30(54,5)$ & $12(21,8)$ & $9(16,4)$ & $4(7,3)$ & - \\
\hline \multirow[t]{2}{*}{ Resolução de Problemas } & b164 & Funções cognitivas superiores & $30(54,5)$ & $12(21,8)$ & $9(16,4)$ & $4(7,3)$ & - \\
\hline & d175 & Resolver Problemas & $12(21,8)$ & $15(27,3)$ & $11(20,0)$ & $14(25,5)$ & $3(5,5)$ \\
\hline Memória & b144 & Funções da memória & $11(20,0)$ & $16(29,1)$ & $7(12,7)$ & $16(29,1)$ & $5(9,1)$ \\
\hline
\end{tabular}

A relação das categorias da MIF está para o segundo qualificador de atividade e participação que corresponde a capacidade (sem ajuda). Dados expressos em frequência.

\section{DISCUSSÃO}

Verificou-se que a maioria dos idosos residentes na ILPI em questão, eram do sexo feminino $(72,7 \%)$, sendo que o diagnóstico dominante foi o de doença de Alzheimer (45,5\%), seguido de Acidente Vascular Encefálico (AVE) (14,5\%). Esses resultados são corroborados por outros estudos, em que mostram a relação da prevalência de idosos do sexo feminino, caracterizando o fenômeno da feminilização da população idosa. ${ }^{13-15}$ Há que se considerar também que, no Brasil, o número de mulheres idosas prevalece sobre o de homens, uma vez que há uma mortalidade diferencial por sexo. ${ }^{16}$

Quanto à faixa etária, a média foi de 79,7 anos; esse dado se assemelha aos achados de Lima et al. ${ }^{7} \mathrm{e}$ Araujo et al., ${ }^{6}$ em que constatou uma média de idade de 
76,5 e 77,9 anos, respectivamente. Também se pode afirmar que esse perfil foi semelhante ao da população brasileira. $^{18}$

A avaliação da memória e capacidade funcional é de extrema importância no processo de envelhecimento, sendo marcador importante no processo de institucionalização de idosos. O baixo nível educacional ou inexistente dos idosos, na população é sócio-demograficamente relevante para desenvolver dependência moderada ou grave, comprometendo a sua funcionalidade..$^{19}$ No presente estudo, em relação ao grau de escolaridade dos participantes, houve predomínio de idosos analfabetos. A alta prevalência do grau de analfabetismo foi observada também por Converso e lartelli, ${ }^{20}$ em que $50,43 \%$ de 115 idosos avaliados apresentaram este grau de instrução. Além disso, um estudo desenvolvido por Oliani et al. ${ }^{21} \mathrm{em}$ ILPI's, revelou progressão no declínio cognitivo e funcional, à medida que os idosos envelhecem.

Em relação à MIF, que avalia-se o quanto o idoso necessita de assistência de outra pessoa ou recurso de adaptação para realizar suas AVD's, mostrou-se uma maior independência completa $(47,3 \%)$, seguido de dependência mínima (23,6\%), assim como no estudo de Murakami e Scattolin (2010), ${ }^{22}$ que observaram uma média elevada do escore da MIF $(103 \pm 15,3)$, indicando pouco comprometimento da independência funcional em 63 idosos institucionalizados. As maiores dificuldades encontrados nesta população foram nos domínios: banhar-se, vestir-se parte superior e inferior, higiene pessoal, subir escadas e déficit da memória, assim como no estudo de Machado et al. ${ }^{23} \mathrm{E}$ o domínio de alimentar-se e arrumar-se foi que mostrou maior independência, indo ao encontro do estudo de Viana et al. ${ }^{24}$ e Greve et al. ${ }^{25}$ podendo-se observar que a alimentação foi a atividade com melhor desempenho para todos as doenças avaliadas. Este bom desempenho no domínio de alimentação, pode ser explicado por ser uma atividade que não requer deslocamento e exige pouco desempenho motor, cognitivo e visual, podendo ser realizada por idosos com diferentes sequelas. ${ }^{26}$

À luz da CIF, pode-se inferir que os idosos avaliados apresentam, em sua maioria, graus significativos de desempenho funcional para atividades analisadas. Referente a isto, podemos observar tais categorias com maior frequência: comer, beber, vestir-se, funções de defecação, mudar a posição do corpo, transferir a própria posição, funções de percepção, comunicação-recepção de mensagens orais e não orais, fala, interações pessoais básicas e funções cognitivas superiores como nenhuma dificuldade, ou seja, qualificador 0 , conforme a ICF (Tabela 3).

Comparando a MIF com a ICF, percebeu-se relação positiva de seus domínios com as categorias desta classificação, assim como seus qualificadores com o escore de tal instrumento. O que é visto no estudo de Fréz et al., ${ }^{7}$ onde relacionaram a MIF com a ICF e demonstraram uma forte concordância entre elas. Além disso, no estudo citado anteriormente, também ocorreu a frequência maior no componente Atividade e Participação. Logo, a MIF demonstrou-se capaz de avaliar o idoso de maneira completa, abordando-o de maneira ampla, tanto no aspecto de capacidade funcional, como no cognitivo, assim como a ICF.

Uma possível limitação deste estudo estaria relacionada ao fato da amostra ter sido de conveniência e de uma única ILPI, o que poderia limitar a generalização dos dados a indivíduos de outras ILPI's e regiões. Entretanto, apesar das limitações, o presente estudo mostra a necessidade de maior atenção aos idosos institucionalizados, tanto por parte de ações governamentais, quanto privadas, a fim de que os idosos tenham melhor manutenção de sua capacidade funcional.

\section{CONSIDERACÕES FINAIS}

Os resultados indicam que os idosos em questão apresentavam independência completa para realizar suas atividades de vida diária. Além disso, observou-se uma forte concordância entre os domínios da MIF e as categorias da ICF, principalmente no componente Atividade e Participação.

A interpretação e discussão dos resultados, por meio da relação do instrumento de avaliação da capacidade funcional com a ICF, possibilitou-nos uma visão mais ampliada sobre o processo de saúde e de envelhecimento de uma população de idosos institucionalizados. Estes dados poderão ser utilizados como indicadores para o desenvolvimento de estratégias e propostas de intervenções que favoreçam a demanda clínica e pessoal destes idosos.

Sugere-se, em estudos futuros, verificar as interferências da institucionalização em ILPI's sobre a funcionalidade de idosos e espera-se que a ICF seja incorporada e utilizada em diversos setores da saúde, inclusive em ILPI's, por equipes multidisciplinares, por meio de uma linguagem unificada e padronizada, proporcionando ações de saúde que contemplem o indivíduo como um todo.

\section{REFERÊNCIAS}

1. Minosso J, Amendola F, Alvarenga M, Oliveira M. Validação, no brasil, do índice de Barthel em idosos atendidos em ambulatórios. Acta Paulista de Enf 2010;2(23):218-23. doi: 10.1590/1982-0194201600050

2. Garcia LC, Malaman AB. Avaliação do medo de quedas e sua correlação com o desempenho funcional, cognitivo e alterações do equilíbrio em idosos de comunidade. Rev. inspirar mov. \& saúde, São Paulo 2015;7(1):6-11.

3. Quintana J, Ferreira E, Santos S, Pelzer M, Lopes M, Barros E. A utilização da classificação internacional de funcionalidade, incapacidade e saúde no cuidado aos idosos. Rev. de Enf. Referência 2014;1:145-52. doi: 10.12707/RIII1292.

4. Depolito C, Leocadio, P, Cordeiro R. Declínio funcional de idosa institucionalizada: aplicabilidade do modelo da classificação internacional de funcionalidade, incapacidade e saúde. Fisioterapia e Pesquisa, São Paulo 2009;2(16):183-9. doi: 10.590/1809-2950/11556021042014.

5. Santos GS, Cunha CK. O. Capacidade funcional e sua mensuração em idosos: uma revisão integrativa. Revista REFACS, São Paulo 2014;2(3):219-29.

6. Spoorenberg S, Reijneveld S, Middel B, Uittenbroek R, Kremer $\mathrm{H}$, Wynia $\mathrm{K}$. The geriatric ICF core set eflecting health related problems in community-living older adults 
aged 75 years and older without dementia: development and validation. Disability and Rehabilitation 2015;44(3):1-7. doi: 10.1080/09638288.2016.1244291

7. Fréz $R$, Vignola $P$, Kaziyama $S$, Spezzano $C$, Filippo $M$, Battistella $R$. The relationship between the functional independence measure and the international classification of functioning, disability, and health core set for stroke, CEP 2013;5716:150. doi: 10.5935/0104-7795.20130005

8. Goldim JR. Manual de Iniciação à Pesquisa em Saúde. 2. ed. Porto Alegre: Da Casa, 2000.

9. Altermann C, Martins S, Carpes P, Mello-Carpes B. Influence of mental practice and movement observation on motor memory, cognitive function and motor performance in the elderly. Braz J Phys Ther 2014;18(2):201-9. doi: 10.1590/ S1413-35552012005000150

10. Lourenço A, Veras R. Mini-Exame do Estado Mental: características psicométricas emidosos ambulatoriais. Revista de Saúde Pública 2006;40(4):712-9.

11. Borges JB. Avaliação da medida de independência funcional - escala MIF - e qualidade de serviço - escala SERVQUAL - em cirurgia cardíaca. Tese (doutorado) - Universidade Estadual Paulista, Faculdade de Medicina de Botucatu, 117 f., 2006.

12. World Health Organization-WHO. International classification of functioning, disability and health: ICF. Geneva: World Health Organization. 2001.

13. Almeida A, Mafra S, Da Silva P, Kanso S. The Feminization of Old Age: a focus on the socioeconomic, personal and family characteristics of the elderly and the social risk. Textos \& Contextos (Porto Alegre) 2015;14(1):115-31. doi: 10.15448/1677-9509.2016.2.25311

14. Campolina G, Adami F, Santos F, Lebrão L. A transição de saúde e as mudanças na expectativa de vida saudável da população idosa: possíveis impactos da prevenção de doenças crônicas. Cad. Saúde Pública 2013;29(6):1217-29. doi: 10.1590/0102-311XED011216

15. Marinho M, Vieira A, Andrade O, De Melo S. Grau de dependência de idosos residentes em instituições de longa permanência. Revista Gaúcha de Enfermagem 2013;34(1):10410. doi: 10.1590/1983-1447.2016.04.57067

16. Araujo B, Moreira B, Villegas P, Loureiro C, Israel V, Gato $\mathrm{S}$. Investigação dos saberes quanto à capacidade funcional e qualidade de vida em idosas institucionalizadas, sob a ótica da CIF. Acta fisiátrica 2015;22(3). doi: 10.5935/01047795.20150022
17. Lima O, Martins J, Gonzaga B, Pinto C, Diógenes G, De Sousa C. Qualidade de vida de idosas institucionalizadas na cidade de Fortaleza/CE. Rev. Saúde Públ. Santa Cat., Florianópolis 2015;8(3):55-66.

18. Instituto Brasileiro de Geografia e Estatística - IBGE. Censo demográfico 2010: características da população e dos domicílios - resultados do universo. IBGE, Rio de Janeiro, 2011.

19. Domiciano, B, Braga P, Silva D, Santos D, Vasconcelos D, Macena M. Cognitive function of elderly residents in longterm institutions: effects of a physiotherapy program. Rev. Bras. de Geriatria e Gerontologia 2016;19(1):57-70. doi: 10.1590/1809-9823.2016.14137

20. Converso M, lartelli I. Caracterização e análise do estado mental e funcional de idosos institucionalizados em instituições públicas de longa permanência. J Bras Psiquiatr 2007;56(4):267-72.

21. OlianiM, ChristofolettiG, StellaF, GobbiB, GobbiS. Locomoção e desempenho cognitivo em idosos institucionalizados com demência. Fisioterapia em movimento 2007;20(1):109-14. doi: 10.1590/1980-5918.029.004.ao01

22. Murakami, L, Scattolin F. Avaliação daindependência funcional e da qualidade de vida de idosos institucionalizados. Rev Medica Herediana 2010;21(1):18-26.

23. Machado N, Machado N, Soares M. Comparação entre a capacidade e desempenho: um estudo sobre a funcionalidade de idosos dependentes. Revista Latino-Americana de Enfermagem 2013;21(6):1321-9. doi: 10.1590/15188345.0000 .2791

24. Viana P, Lourenço C, Oliveira F, Resende M. Medida de independência funcional nas atividades de vida diária em idosos com sequela de acidente vascular encefálico no Complexo Gerontológico Sagrada Família em Goiânia. Rev Bras Geriatr Gerontol 2008;11(1):1809-23. doi: 10.1590/1809-9823.2016.14137

25. Greve P, Guerra G, Portela A, Portes S, Rebelatto R. Correlações entre mobilidade e independência funcional em idosos institucionalizados e não institucionalizados. Fisioter Mov 2007;20(4):117-24. doi: doi: 10.1590/19805918.029.004.ao01

26. Dantas L, Bello A, Barreto L, Lima S. Capacidade funcional de idosos com doenças crônicas residentes em Instituições de Longa Permanência. Revista Brasileira de Enfermagem 2013;66(6):914.

Como citar: FLEIG, Tania Cristina Malezan; OLIVEIRA, Murilo Rezende. Avaliação da funcionalidade de idosos institucionalizados: relação entre a MIF e a ICF. Cinergis, Santa Cruz do Sul, v. 18, n. 3, maio 2017. ISSN 2177-4005. Disponível em: < https:// online.unisc. br/seer/index.php/cinergis/article/view/8902 >. Acesso em: 22 jun. 2017. doi:http://dx.doi.org/10.17058/cinergis. v18i3.8902. 